\title{
The decline of the bumble bees and cuckoo bees (Hymenoptera: Apidae: Bombini) of Western and Central Europe
}

\author{
Andrzej Kosior, Waldemar Celary, Paweł Olejniczak, Jan Fijał, Wiesław Król, Wojciech Solarz and Piotr Płonka
}

\begin{abstract}
The bumble and cuckooo bees (Hymenoptera: Apidae: Bombini; Bombus spp. and Psithyrus spp., respectively) are important plant pollinators and any decline in numbers or species constitutes a significant threat both to biological diversity and to whole economies. The distribution, status and factors threatening all 60 known taxa (species and subspecies) of Bombini of 11 countries of Western and Central Europe (Belgium, the Netherlands, Luxembourg, Denmark, Germany, Switzerland, Austria, Czech Republic, Slovakia, Hungary, Poland) were assessed from the beginning of the 20th century. The analysis was based on a literature review, unpublished data, personal communications, our own observations, and an expert review. The IUCN Red List categories were used for assessing the threat of extinction. Eighty per cent of taxa were threatened in at least one country of the region, and $30 \%$ of taxa were threatened throughout their range
\end{abstract}

in the countries considered. More species went extinct per country in the second than in the first half of the 20th century, and four taxa went extinct in all 11 countries during 1951-2000. Amongst the factors adversely affecting the Bombini anthropogenic factors (particularly those associated with large-scale farming schemes) appear to be of greater importance than natural factors. To halt population declines and species extinctions it will be necessary to preserve aspects of traditional farming practices and for all Bombini to be afforded legal protection in all countries of the region. The implementation of the European Union's Common Agricultural Policy is likely to have the greatest single impact upon pollinators in the near future.

Keywords Agriculture, Apidae, bees, Bombini, Bombus, Europe, Hymenoptera, pollinator loss, Psithyrus, threats.

\section{Introduction}

Because of their role as pollinators the bumble and cuckoo bees (Hymenoptera: Apidae: Bombini; Bombus spp. and Psithyrus spp., respectively) have been receiving increasing attention, and a marked decrease in numbers has been noted worldwide (Williams, 1982, 1988, 1989, 2005; Corbet et al., 1991; Monsevičius, 1995; Allen-Wardell et al., 1998; Kearns et al., 1998; Goulson, 2003; Goulson et al., 2005). Pollinators play a key role in ecosystems, ensuring production value in crops, survival and maintenance of plant diversity, and trophic integrity (Herrera \& Pellmyr, 2002). An estimated $>80 \%$ of crops in the European Union directly depend upon biotic pollination (Williams, 1994). Similarly, $>80 \%$ of all wild plant species depend on insect pollination

\footnotetext{
Andrzej Kosior (Corresponding author), Paweł Olejniczak, Jan Fijał, Wiesław Król, Wojciech Solarz and Piotr Płonka Institute of Nature Conservation, Polish Academy of Sciences, Mickiewicza 33, 31-120 Kraków, Poland. E-mail kosior@iop.krakow.pl

Waldemar Celary Institute of Systematics and Evolution of Animals, Sławkowska 17, 31-016 Kraków, Poland.

Received 19 November 2004. Revision requested 17 March 2005. Accepted 19 September 2005
}

and $>60 \%$ of plant species show pollination limitation (Burd, 1994). The drop in the numbers of insect pollinators, particularly Bombini, constitutes a significant threat both to biological diversity and to whole economies.

In most regions of the world, however, there is a significant lack of data on the biology, distribution, status or causes of the decline of the Bombini. This is the case even in Europe with its relatively long tradition of entomological studies. Here we assess the distribution, status and factors threatening all known taxa of Bombini in 11 Western and Central European countries, and assess the implications of the decline in these taxa for agriculture and biodiversity.

\section{Methods}

We assessed the Bombini of Belgium, the Netherlands, Luxembourg, Denmark, Germany, Switzerland, Austria, Czech Republic, Slovakia, Hungary and Poland (Fig. 1). The evaluation of species composition and ranges were based on a literature review, unpublished data, and our own observations. A significant part of this information, as well as the degree of threat to individual species and the factors adversely affecting them, was obtained from 


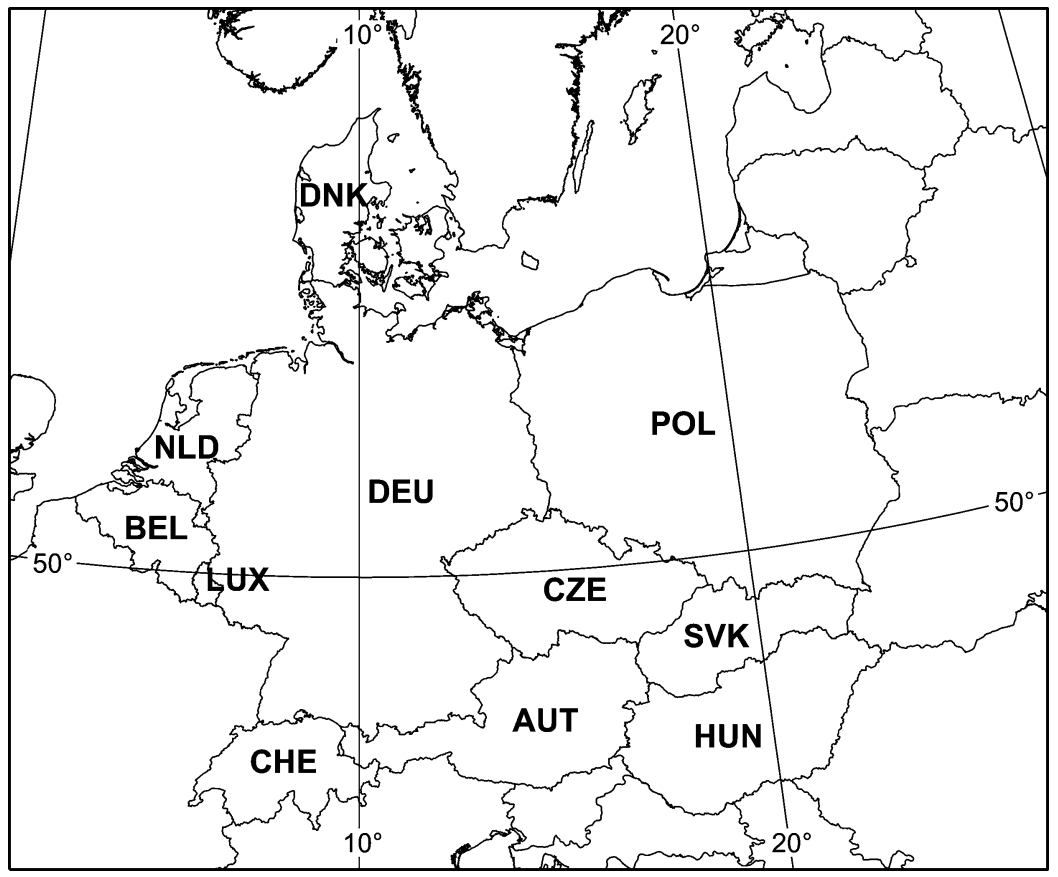

Fig. 1 The 11 countries of Western and Central Europe for which information on Bombini was collated: BEL, Belgium; NLD, the Netherlands; LUX, Luxembourg; DNK, Denmark; DEU, Germany; CHE, Switzerland; AUT, Austria; CZE, Czech Republic; SVK, Slovakia; HUN, Hungary; POL, Poland.

questionnaires completed by experts from the countries covered by the analysis.

For assessing the threat of extinction we used the IUCN Red List categories (IUCN, 2001). For each taxon (species or subspecies) an index of overall threat was also calculated by dividing the number of countries in which the species was in one of the Red List categories (EX, Extinct; CR, Critically Endangered; EN, Endangered; VU, Vulnerable; NT, Near Threatened) by the total number of countries in which the species was present.

To test whether there has been an increase in threats to the Bombini the 20th century was divided into preand post-1950 periods and the total number of extinction events in all countries compared for these two periods. A list of 21 anthropogenic and natural factors adversely affecting the Bombini was prepared from a literature review. The experts, to whom this list was sent, chose factors that they considered to be drivers of Bombini decline in their countries. Systematics of the Bombini followed Reinig (1981), von Hagen \& Aichorn (2003), Přidal (2004) and NHM (2005).

Data were obtained from the following sources: Belgium: Rasmont \& Mersch (1988), Rasmont et al. (1993), Rasmont (1995), Rasmont et al. (1995), P. Rasmont (pers. comm., 2002, 2004). Netherlands: Warncke (1986), Peeters et al. (1999), C. Achterberg (pers. comm., 2002). Luxembourg: Warncke (1986), Rasmont et al. (1995),
P. Rasmont (pers. comm., 2002). Denmark: I. Calabuig (pers. comm., 2004), H.B. Madsen (pers. comm., 2004), H.B. Madsen \& R. Mortensen. (unpubl. data). Germany: Riess et al. (1976), Wergin (1977), Warncke et al. (1984), Warncke (1986), Westrich (1990), Schwartz et al. (1996), Westrich et al. (1998), van der Smissen \& Rasmont (1999), von Hagen \& Aichhorn (2003). Switzerland: Warncke (1986), F. Amiet (pers. comm., 1994, 2004), Rasmont et al. (1995), von Hagen \& Aichhorn (2003), S. Durrer (pers. comm., 2004). Austria: Aichorn (1983), Kosior (1992a), Schwartz et al. (1996), Neumayer \& Paulus (1999), J. Neumayer (pers. comm., 2002), von Hagen \& Aichhorn (2003). Czech Republic: May (1959), Tkalců (1974, 1999), Pagliano (1994), M. Pavelka (pers. comm., 2002), I. Valterova (pers. comm., 2002), Přidal \& Tkalců (2003), Přidal (2004), A. Přidal (pers. comm., 2004). Slovakia: May (1959), Tkalců (1974), Beláková et al. (1979), Pagliano (1994), Lukas (2001), R. Chlebo (pers. comm., 2002, 2004), J. Slamecka (pers. comm., 2002), Přidal \& Tkalců (2003), Pridal (2004), V. Ptáček (pers. comm., 2004), V. Smetana (pers. comm., 2004). Hungary: Móczár (1957), Rasmont (1983), K. Szauter (pers. comm., 2001), M. Sárospataki (pers. comm., 2002), Sárospataki et al. (2003). Poland: Kosior (1987, 1990, 1992c), Kosior \& Nosek (1987), Banaszak (1993, 1996, 2002), Dylewska (1996), Kosior \& Witkowski (1997), Celary et al. (2004), T. Pawlikowski (unpubl. data), Starzyk \& Kosior (1985). 


\section{Results}

The earliest information on Bombini for the countires considered dates from the last half of the 19th century, from Poland (Siła-Nowicki, 1864; Wierzejski, 1868, 1874; Śnieżek 1893, 1899) and Austria (Müller, 1881). There are records for the first half of the 20th century from Belgium (Rasmont \& Mersch, 1988), Denmark (H.B. Madsen \& R. Mortensen, unpubl. data) and the Netherlands (C. Achterberg, pers. comm., 2002), and later from Hungary (Móczár, 1957), the area of the Czech Republic and Slovakia (May, 1959) and the remaining countries of Western and Central Europe considered here. The information pertained mainly to records of taxa, and only to a limited extent to distribution, numbers and habitats.

The 60 taxa (species and subspecies) of Bombini found to occur in the 11 countries since the beginning of the 20th century included 48 taxa of bumble bees and 12 taxa of cuckoo bees (Table 1). The least number of taxa (26-30) were found in small (Luxembourg) and lowland countries (the Netherlands and Denmark). Countries with diverse physical and geographical features (Germany, Switzerland, Austria, Czech Republic, Slovakia and Poland), had the most taxa (38-45). There was no relation between country area and the number of taxa.

The first intimation of threats to the Bombini, and of known extinctions, dates from the 1940s for Belgium (Rasmont et al., 1993), Denmark (H.B. Madsen \& R. Mortensen, unpubl. data), the Netherlands (C. Achterberg, pers. comm., 2002) and Poland (Banaszak, 2000). Extinctions also subsequently occurred in Austria (Aichhorn, 1983; J. Neumayer, pers. comm., 2002), Switzerland (Amiet, 1994), Hungary (M. Sárospataki, pers. comm., 2002), Germany (Riess et al., 1976), and the Czech Republic (A. Přidal, pers. comm., 2004).

We were able to evaluate the status of most taxa in the 11 countries but were not able to evaluate $74 \%$ of the taxa occurring in Luxembourg. Data Deficient species accounted for $<10 \%$ of taxa except for Poland $(26.8 \%)$ and Slovakia (13.2\%; Table 2). Of the taxa occurring in the whole study area, $16(26.7 \%)$ were categorized as CR, $22(36.7 \%)$ as EN, $38(63.3 \%)$ as VU and $38(63.3 \%)$ as NT in at least one country. Thirty-two taxa $(53.3 \%)$ were categorized as EX, CR, EN, VU or NT in $>75 \%$ of the countries in which they occurred. This includes 19 $(31.7 \%)$ taxa threatened throughout their range in the 11 countries. Only 11 taxa (18.3\%) were not categorized as EX, CR, EN, VU or NT in $>75 \%$ of the countries in which they occurred. Our analysis may, however, underestimate the overall threat to those species that have only been recorded in one or two countries.

The greatest number of taxa categorized as EX, CR, EN, VU or NT were found in Switzerland (35), Czech
Republic (29), Germany and Austria (28 each), and the lowest in Poland (15) and Slovakia (16). When the percentages of threatened taxa were considered, the most threatened are in Switzerland (79.5\%), Hungary (78.1\%), Czech Republic (74.4\%) and Belgium (72.2\%), and the least in Slovakia (42.1\%) and Poland (36.6\%). Correlations between the percentage of species categorized as EX, threatened (CR, EN, VU) or NT in each country, both separately and pooled, and country area were insignificant.

Six and 13 taxa, respectively, became extinct in at least one country in the periods 1901-1950 and 1951-2000 (Table 3). Four species became totally extinct in the 11 countries (B. armeniacus, B. cullumanus, B. serrisquama and B. sidemii) in 1951-2000. Of 29 country extinctions 21 (72.4\%) occurred in 1951-2000, significantly more than in 1901-1950 ( $\chi^{2}=5.533$, df $\left.=1, \mathrm{P}<0.05\right)$. All first records of Bombini occurred in the second half of the 20th century (Table 3).

In the opinion of experts the reason for changes in composition and distribution of Bombini in the 11 countries can be attributed to the effects of a range of both anthropogenic and natural factors (Table 4). Of the anthropogenic factors, fragmentation and decrease in suitable habitats were considered to have adversely affected Bombini in 10 of the 11 countries, and homogenization of habitats due to agricultural practices and intensive use of chemical fertilizers, herbicides and pesticides, to have affected Bombini in nine countries. Pollution by heavy metals, mortality due to tourism and road traffic, and expansion of urban areas were considered to be important drivers of pollinator loss in approximately half of the countries examined.

Changes adversely affecting the Bombini in the 11 countries have in some cases resulted in legal protection and some species have been included in Red Data lists (Table 5). In Germany, Switzerland, Czech Republic, Slovakia and Poland all or almost all Bombini taxa are legally protected. In Belgium, Austria and Hungary selected species are protected. The Bombini are not legally protected in the Netherlands, Luxembourg or Denmark.

\section{Discussion}

Although the use of a data set compiled by country is to some extent artificial (international boundaries generally being arbitrary rather than following natural landscape features) our analyses yielded some important insights for conservation and confirmed concerns about the conservation status of this group of pollinators. Only $20 \%$ of the 60 Bombini taxa were not threatened in some way in the 11 countries studied. The remaining $80 \%$ were threatened in at least one country, with more than 
Table 1 Red List categories (IUCN, 2001) ${ }^{1}$ of the 60 Bombini taxa (species and subspecies) of 11 Western and Central European countries (Fig. 1), with their threat index (see text for further details).

\begin{tabular}{|c|c|c|c|c|c|c|c|c|c|c|c|c|}
\hline \multirow[b]{2}{*}{ Taxon } & \multicolumn{11}{|c|}{ Countries } & \multirow{2}{*}{$\begin{array}{l}\text { Threat } \\
\text { index }\end{array}$} \\
\hline & BEL & NLD & LUX & DNK & DEU & CHE & AUT & CZE & SVK & HUN & POL & \\
\hline Bombus alpinus ${ }^{2} \mathrm{~L}$. & & & & & EX & EN & NT & & & & & 1.00 \\
\hline Bombus argillaceus (Scop.) & & & & & EN & VU & VU & & LC & VU & & 0.80 \\
\hline Bombus armeniacus (Rad.) & & & & & & & EX & EX & & & & 1.00 \\
\hline Bombus brodmannicus (Vogt) & & & & & & NT & & & & & & 1.00 \\
\hline Bombus confusus Schenck & EX & EX & NE & & EN & EX & $\mathrm{CR}$ & CR & EN & NT & VU & 0.90 \\
\hline Bombus cryptarum Fabr. & VU & & VU & NT & $\mathrm{DD}$ & VU & NT & VU & $\mathrm{DD}$ & & $\mathrm{DD}$ & 0.67 \\
\hline Bombus cullumanus (K.) & EX & EX & & EX & EX & & & & & & & 1.00 \\
\hline Bombus distinguendus Mor. & EX & CR & & VU & EN & EX & EX & $\mathrm{CR}$ & $\mathrm{CR}$ & EX & VU & 1.00 \\
\hline Bombus fragrans ${ }^{2}$ Pall. & & & & & & & EX & EX & DD & EN & EX & 0.80 \\
\hline Bombus gerstaeckeri ${ }^{2}$ Mor. & & & & & VU & VU & NT & & & VU & & 1.00 \\
\hline Bombus haematurus (Kriech.) & & & & & & & & & & VU & & 1.00 \\
\hline Bombus hortorum (L.) & VU & LC & NE & $\mathrm{LC}$ & LC & LC & LC & NT & LC & LC & LC & 0.18 \\
\hline Bombus humilis Ill. & EN & NT & $\mathrm{NE}$ & NT & NT & VU & VU & $\mathrm{EN}$ & $\mathrm{LC}$ & $\mathrm{LC}$ & VU & 0.73 \\
\hline Bombus hypnorum (L.) & LC & LC & NE & $\mathrm{LC}$ & $\mathrm{LC}$ & $\mathrm{LC}$ & LC & CR & VU & VU & LC & 0.27 \\
\hline Bombus inexspectatus ${ }^{2}$ (Tkalcu) & & & & & & VU & EN & & & & & 1.00 \\
\hline Bombus jonellus ${ }^{3}(\mathrm{~K})$. & EN & NT & NE & NT & VU & EN & VU & VU & DD & & VU & 0.80 \\
\hline Bombus laesus mocsaryi ${ }^{2}$ Kriechb. & & & & & & NT & EX & & $\mathrm{CR}$ & VU & VU & 1.00 \\
\hline Bombus lapidarius (L.) & VU & LC & NE & $\mathrm{LC}$ & $\mathrm{LC}$ & $\mathrm{LC}$ & $\mathrm{LC}$ & LC & $\mathrm{LC}$ & LC & LC & 0.09 \\
\hline Bombus lucorum (L.) & VU & & VU & LC & $\mathrm{LC}$ & $\mathrm{LC}$ & $\mathrm{LC}$ & $\mathrm{LC}$ & $\mathrm{LC}$ & NT & LC & 0.30 \\
\hline Bombus maculidorsis Skor. & & & & & & & & & & & VU & 1.00 \\
\hline Bombus magnus Vogt & VU & & VU & NT & EN & NT & NT & VU & & & DD & 0.88 \\
\hline Bombus mendax ${ }^{2}$ (Gerst.) & & & & & VU & VU & NT & & & & & 1.00 \\
\hline Bombus mesomelas ${ }^{2}$ Gerst. & NE & & & & EN & VU & NT & $\mathrm{CR}$ & DD & EX & DD & 0.63 \\
\hline Bombus monticola hypsophilus ${ }^{2}$ (Skor.) & & & & & NT & NT & NT & & & & & 1.00 \\
\hline Bombus mucidus² (Gerst.) & & & & & VU & VU & NT & & & & & 1.00 \\
\hline Bombus muscorum (L.) & VU & NT & $\mathrm{NE}$ & NT & EN & NT & CR & CR & EN & NT & LC & 0.82 \\
\hline Bombus pascuorum (Scop.) & LC & LC & NE & LC & LC & $\mathrm{LC}$ & LC & LC & LC & LC & LC & 0.00 \\
\hline Bombus pomorum (Panz.) & EX & EX & & EX & EN & $\mathrm{EN}$ & $\mathrm{CR}$ & $\mathrm{CR}$ & $\mathrm{CR}$ & NT & VU & 1.00 \\
\hline Bombus pratorum (L.) & LC & $\mathrm{LC}$ & $\mathrm{NE}$ & LC & LC & $\mathrm{LC}$ & LC & LC & LC & NT & LC & 0.09 \\
\hline Bombus pyrenaeus afasciatus Dyl. & & & & & & & & & $\mathrm{LC}$ & & DD & 0.00 \\
\hline Bombus pyrenaeus tenuifasciatus ${ }^{2}$ Vogt & & & & & NT & NT & LC & & & & & 0.67 \\
\hline Bombus ruderarius (Müll.) & VU & NT & NE & NT & VU & VU & VU & EN & LC & LC & LC & 0.64 \\
\hline Bombus ruderatus ruderatus (Fabr.) & CR & CR & & EX & NT & EN & & CR & VU & NT & VU & 0.50 \\
\hline Bombus ruderatus eurynotus (D. T.) & & & NE & & & & NT & & & & & 1.00 \\
\hline Bombus schrencki (Mor.) & & & & & & & & & & & DD & 0.00 \\
\hline Bombus semenoviellus ${ }^{4}$ Skor. & & & & & LC & & & DD & & & DD & 0.00 \\
\hline Bombus serrisquama² (Mor.) & & & & & & & & & & EX & & 1.00 \\
\hline Bombus sichelii sichelii (Rad.) & & & & & & & & DD & DD & & EX & 0.33 \\
\hline Bombus sichelii alticola ${ }^{2}$ (Kriechb.) & & & & & VU & VU & $\mathrm{LC}$ & & & & & 0.67 \\
\hline Bombus sidemii (Rad.) & & & & & & & & EX & & & & 1.00 \\
\hline Bombus soroeensis proteus Gerst. & EN & EN & NE & NT & NT & EN & $\mathrm{LC}$ & VU & VU & VU & VU & 0.82 \\
\hline Bombus soroeensis soroeensis (Fabr.) & EN & & & NT & NT & & & VU & VU & VU & VU & 1.00 \\
\hline Bombus subterraneus (L.) & CR & CR & NE & VU & NT & EN & CR & CR & EN & VU & VU & 0.91 \\
\hline Bombus sylvarum (L.) & VU & EX & NE & NT & NT & VU & NT & $\mathrm{CR}$ & LC & LC & LC & 0.64 \\
\hline Bombus terrestris (L.) & VU & LC & VU & LC & $\mathrm{LC}$ & $\mathrm{LC}$ & $\mathrm{LC}$ & $\mathrm{LC}$ & $\mathrm{LC}$ & $\mathrm{LC}$ & LC & 0.18 \\
\hline Bombus veteranus (Fabr.) & EN & VU & NE & VU & VU & VU & EN & CR & CR & & VU & 0.90 \\
\hline Bombus wurflenii mastrucatus ${ }^{2}$ Gerst. & $\mathrm{DD}$ & & & & NT & EN & $\mathrm{LC}$ & VU & $\mathrm{LC}$ & & DD & 0.43 \\
\hline Bombus zonatus² (Smith) & & & & & & & & & & $\mathrm{CR}$ & & 1.00 \\
\hline Psithyrus barbutellus (Kirby) & EN & EN & EN & VU & LC & NT & LC & CR & VU & NT & NT & 0.82 \\
\hline Psithyrus bohemicus (Seidl.) & LC & LC & NE & $\mathrm{LC}$ & LC & NT & LC & LC & $\mathrm{LC}$ & VU & LC & 0.18 \\
\hline Psithyrus campestris (Panz.) & VU & $\mathrm{LC}$ & $\mathrm{NE}$ & VU & $\mathrm{LC}$ & $\mathrm{LC}$ & $\mathrm{LC}$ & NT & $\mathrm{LC}$ & VU & LC & 0.36 \\
\hline Psithyrus flavidus flavidus (Evers.) & & & & & & & & & & & DD & 0.75 \\
\hline Psithyrus flavidus alpium² (Rich.) & & & & & NT & NT & NT & & & & DD & 0.00 \\
\hline Psithyrus maxillosus (Klug) & & & & & & NT & VU & CR & VU & & & 1.00 \\
\hline Psithyrus norvegicus Sp.-Schn. & DD & LC & NE & NT & LC & NT & NT & EN & VU & & DD & 0.50 \\
\hline Psithyrus quadricolor quadricolor Lep. & $\mathrm{DD}$ & & & EX & NT & & & CR & CR & & DD & 0.83 \\
\hline Psithyrus quadricolor meridionalis (Rich.) & & & DD & & NT & NT & NT & $\mathrm{CR}$ & $\mathrm{CR}$ & & & 0.67 \\
\hline Psithyrus rupestris (Fabr.) & EN & NT & EN & LC & LC & EN & LC & LC & LC & NT & LC & 0.45 \\
\hline
\end{tabular}


Table 1 (Continued)

\begin{tabular}{|c|c|c|c|c|c|c|c|c|c|c|c|c|}
\hline \multirow[b]{2}{*}{ Taxon } & \multicolumn{11}{|c|}{ Countries } & \multirow{2}{*}{$\begin{array}{l}\text { Threat } \\
\text { index }\end{array}$} \\
\hline & BEL & NLD & LUX & DNK & DEU & $\mathrm{CHE}$ & AUT & CZE & SVK & HUN & POL & \\
\hline Psithyrus sylvestris Lep. & LC & LC & NE & LC & LC & LC & LC & VU & LC & VU & LC & 0.18 \\
\hline Psithyrus vestalis (Geoff. in Four.) & VU & $\mathrm{LC}$ & NE & $\mathrm{LC}$ & LC & VU & $\mathrm{LC}$ & $\mathrm{LC}$ & $\mathrm{LC}$ & NT & $\mathrm{LC}$ & 0.27 \\
\hline Total no. of taxa & 33 & 26 & 27 & 30 & 44 & 44 & 45 & 39 & 38 & 32 & 41 & \\
\hline
\end{tabular}

${ }^{1}$ EX, Extinct; CR, Critically Endangered; EN, Endangered; VU, Vulnerable; NT, Near Threatened; LC, Least Concern; DD, Data Deficient; NE, Not Evaluated. Note that LC is not actually a category on the Red List but signifies that the species has been assessed and found not to be threatened.

${ }^{2}$ Taxa with specific habitat requirements (alpine, mountain or steppe habitat)

${ }^{3} \mathrm{~A}$ glacial relict in the mountains of Central Europe

${ }^{4}$ Species expanding its range westwards

Table 2 Number and percentage (in parentheses) of Bombini taxa (Table 1) in each Red List Category (IUCN, 2001) of 11 Western and Central European countries (Fig. 1).

\begin{tabular}{|c|c|c|c|c|c|c|c|c|c|c|c|}
\hline \multirow[b]{2}{*}{ Category* } & \multicolumn{11}{|c|}{ Countries } \\
\hline & BEL & NLD & LUX & DNK & DEU & CHE & AUT & CZE & SVK & HUN & POL \\
\hline EX & $4(12.1)$ & $4(15.4)$ & 0 & $4(13.3)$ & $2(4.5)$ & $2(4.5)$ & $4(8.9)$ & $3(7.7)$ & 0 & $3(9.4)$ & $2(4.8)$ \\
\hline $\mathrm{CR}$ & $2(6.1)$ & $3(11.5)$ & 0 & 0 & 0 & 0 & $4(8.9)$ & $14(35.9)$ & $6(15.8)$ & $1(3.1)$ & 0 \\
\hline EN & $7(21.2)$ & $2(7.7)$ & $2(7.4)$ & 0 & $7(15.9)$ & $8(18.2)$ & $2(4.4)$ & $3(7.7)$ & $3(7.9)$ & $1(3.1)$ & 0 \\
\hline VU & $11(33.3)$ & $1(3.8)$ & $4(14.8)$ & $5(16.7)$ & $7(15.9)$ & $13(29.5)$ & $5(11.1)$ & $7(17.9)$ & $7(18.4)$ & $11(34.4)$ & $12(29.2)$ \\
\hline NT & 0 & $5(19.2)$ & 0 & 10(33.3) & $12(27.3)$ & $12(27.3)$ & $13(28.9)$ & $2(5.1)$ & 0 & $9(28.1)$ & $1(2.4)$ \\
\hline LC & $5(15.2)$ & $11(42.3)$ & 0 & $11(36.7)$ & $15(34.1)$ & $9(20.5)$ & $17(37.8)$ & $8(20.5)$ & 17(44.7) & $7(21.9)$ & 15(36.6) \\
\hline $\mathrm{DD}$ & $3(9.1)$ & 0 & $1(3.7)$ & 0 & $1(2.3)$ & 0 & 0 & $2(5.1)$ & 5(13.2) & 0 & $11(26.8)$ \\
\hline $\mathrm{NE}$ & $1(3.0)$ & 0 & $20(74.1)$ & 0 & 0 & 0 & 0 & 0 & 0 & 0 & 0 \\
\hline Total & 33 & 26 & 27 & 30 & 44 & 44 & 45 & 39 & 38 & 32 & 41 \\
\hline
\end{tabular}

*See footnote to Table 1

Table 3 Extinctions and first records of Bombini taxa (Table 1) of 11 Western and Central European countries (Fig. 1).

\begin{tabular}{|c|c|c|c|c|}
\hline \multirow[b]{2}{*}{ Taxon } & \multicolumn{2}{|l|}{ Extinction } & \multicolumn{2}{|l|}{ First record } \\
\hline & Before 1950 & After 1951 & Before 1950 & After 1951 \\
\hline B. alpinus & & DEU & & \\
\hline B. armeniacus & & AUT, CZE & & \\
\hline B. confusus & NLD & BEL, CHE & & \\
\hline B. cryptarum & & & & CZE, SVK \\
\hline B. cullumanus & BEL, DNK & DEU, NLD & & \\
\hline & & AUT, BEL, CHE, DNK, & & \\
\hline B. distinguendus & & HUN & & \\
\hline B. fragrans & POL & AUT, CZE & & \\
\hline B. laesus mocsaryi & & AUT & & \\
\hline B. magnus & & & & CZE \\
\hline B. mesomelas & & HUN & & \\
\hline B. pomorum & NLD, DNK & BEL & & \\
\hline B. ruderatus ruderatus & & DNK & & \\
\hline B. semenoviellus & & & & DEU, POL, CZE \\
\hline B. serrisquama & & HUN & & \\
\hline B. sichelii sichelii & POL & & & CZE, SVK \\
\hline B. sidemii & & CZE & & \\
\hline B. sylvarum & & NLD & & \\
\hline P. quadricolor quadricolor & DNK & & & \\
\hline Total no. of taxa & 6 & 13 & 0 & 4 \\
\hline Total no. of extinctions/first records & 8 & 21 & 0 & 8 \\
\hline
\end{tabular}


Table 4 Occurrence of 16 anthropogenic and five natural factors (see text for details) threatening the Bombini (Table 1) of 11 Western and Central European countries (Fig. 1), ranked by the number of countries in which the threats occur.

\begin{tabular}{|c|c|c|c|c|c|c|c|c|c|c|c|c|}
\hline \multirow[b]{2}{*}{ Factors } & \multicolumn{11}{|c|}{ Country } & \multirow{2}{*}{$\begin{array}{l}\text { No. of } \\
\text { countries }\end{array}$} \\
\hline & BEL & NLD & LUX & DNK & DEU & CHE & AUT & CZE & SVK & HUN & POL & \\
\hline \multicolumn{13}{|l|}{ Anthropogenic } \\
\hline $\begin{array}{l}\text { Fragmentation \& decrease in suitable habitats } \\
\text { (including melioration of wetlands) }\end{array}$ & + & + & & + & + & + & + & + & + & + & + & 10 \\
\hline $\begin{array}{l}\text { Homogenization of habitats due to agricultural } \\
\text { practices }\end{array}$ & + & + & & + & + & + & + & + & + & & + & 9 \\
\hline $\begin{array}{l}\text { Intensive use of chemical fertilizers, herbicides } \\
\text { \& pesticides }\end{array}$ & + & + & & + & + & + & + & + & + & & + & 9 \\
\hline $\begin{array}{l}\text { Shortage of food due to frequent mowing } \\
\text { of meadows }\end{array}$ & + & & & + & + & + & + & + & + & & & 7 \\
\hline $\begin{array}{l}\text { Shortage of food due to competition with } \\
\text { honey bees }\end{array}$ & + & & & + & + & & + & & + & & + & 6 \\
\hline Pollution with heavy metals & & + & & & + & & + & + & & + & + & 6 \\
\hline Trampling due to tourism & & & & + & + & & + & + & & + & + & 6 \\
\hline Mortality caused by cars & & & & + & + & & + & + & & + & + & 6 \\
\hline $\begin{array}{l}\text { Burning of old grass in the spring \& stubble } \\
\text { in summer }\end{array}$ & & & & + & & & + & & + & + & + & 5 \\
\hline $\begin{array}{l}\text { Afforestation on xerothermic swards, } \\
\text { meadows \& glades }\end{array}$ & + & & & + & + & & & & + & & + & 5 \\
\hline Expansion of urban areas & + & & & & + & & + & & + & & + & 5 \\
\hline $\begin{array}{l}\text { Destruction of nests by heavy machines on } \\
\text { meadows, fields \& forests }\end{array}$ & & & & & + & & + & & + & & + & 4 \\
\hline Import of alien species for pollination & & & & & + & & + & & + & & + & 4 \\
\hline Pollution by acid rain & & & & & + & & + & + & + & & & 4 \\
\hline Building of dams & & & & & & & & + & & & + & 2 \\
\hline Collecting for museum \& private collections & & & & & & & & & & & + & 1 \\
\hline Natural & & & & & & & & & & & & \\
\hline $\begin{array}{l}\text { Natural succession of forest glades, meadows } \\
\text { \& xerothermic swards }\end{array}$ & & & & + & + & & & + & + & & + & 5 \\
\hline Sterilization of wintering females by & & & & & & & & & & & & 5 \\
\hline Sphaerularia bombi & & & & + & + & & + & & + & & + & \\
\hline $\begin{array}{l}\text { Parasitism or predation by Arachnidae, } \\
\text { Lepidoptera, Diptera, Hymenoptera, \& birds }\end{array}$ & & & & & & & & & & & & 4 \\
\hline \& mammals & & & & & + & + & + & & & & + & \\
\hline $\begin{array}{l}\text { Adverse weather conditions (long winters, } \\
\text { drought) }\end{array}$ & & & & & + & & + & & + & & + & 4 \\
\hline Natural mortality of wintering females & & & & & & & & & & & + & 1 \\
\hline
\end{tabular}

half of all taxa threatened in most countries. This evidence of adverse changes in the species composition and distribution of the Bombini across this large area of Western and Central Europe corroborates the results of country-based studies in this region (Riess et al., 1976; Wergin, 1977; Aichhorn, 1983; Warncke et al., 1984; Starzyk \& Kosior, 1985; Kosior, 1987, 1990, 1992c; Kosior \& Nosek, 1987; Westrich, 1990; Kosior \& Witkowski, 1997; Banaszak et al. 1998; C. Achterberg, pers. comm., 2002; R. Chlebo, pers. comm., 2002; J. Neumayer, pers. comm., 2002; P. Rasmont, pers. comm., 2002; M. Sárospataki, pers. comm., 2002; von Hagen \& Aichhorn, 2003; Celary et al., 2004; S. Durrer, pers. comm., 2004; H.B. Madsen, pers. comm., 2004; A. Přidal, pers. comm., 2004). Neither the total number of taxa nor the total number of threatened species of Bombini per country could be explained by country area or longitude.

The apparently greater number of species extinctions in the second half of the 20th century could be affected by the fact that earlier data may have been less precise. However, a similar trend in increasing extinctions has been noted for butterflies in Poland (Razowski, 1985) and bumble bees in Britain (Williams, 1982). In the second half of the 20th century more species also expanded their ranges than in the pre-1950 period. These expansions do not seem to be facilitated by weaker competition from declining species, as they do not share the same niche, and the species expanding their ranges are probably more tolerant of anthropogenic pressures. 
Table 5 Legal protection accorded to the Bombini of 11 Western and Central European countries (Fig. 1), with their coverage in Red Books and Red Lists of threatened species.

\begin{tabular}{|c|c|c|c|}
\hline Country & Legal protection & Red Book* & Red List* \\
\hline BEL & $\begin{array}{l}\text { Only } 6 \text { species of Bombus, since } 2002 \\
\text { (P. Rasmont, pers. comm., 2002) }\end{array}$ & & \\
\hline NLD & & & $\begin{array}{l}\text { Peeters \& Reemer (2003; does not } \\
\text { include Bombus) }\end{array}$ \\
\hline \multicolumn{4}{|r|}{ 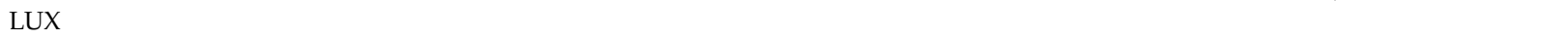 } \\
\hline \multicolumn{4}{|l|}{ DNK } \\
\hline DEU & $\begin{array}{l}\text { All Bombini species since } 1980 \\
\text { (Gauld et al., 1990; von Hagen \& } \\
\text { Aichhorn, 2003) }\end{array}$ & Blab et al. (1984) & $\begin{array}{l}\text { Blab et al. (1984), Westrich \& Schmidt } \\
\text { (1985), Winter (1994), Westrich et al. } \\
\text { (1998), Dathe \& Saure (2000) }\end{array}$ \\
\hline CHE & $\begin{array}{l}\text { Only the Vaud Canton protects all } \\
\text { useful insects, including bumble } \\
\text { bees (Gauld et al., 1990) }\end{array}$ & & Amiet, 1994 \\
\hline AUT & $\begin{array}{l}\text { None, except for Burgenland, where } \\
\text { catching any useful insects, } \\
\text { including Bombini, is prohibited } \\
\text { by law }\end{array}$ & $\begin{array}{l}\text { J. Gepp (1983; includes only } \\
\text { B. inexpectatus in Carinthia) }\end{array}$ & $\begin{array}{l}\text { Aichorn (1983), von Hagen \& Aichorn } \\
\text { (2003) (bumble bees \& threats thereto } \\
\text { are only referred to in a comment) }\end{array}$ \\
\hline CZE & $\begin{array}{l}\text { All Bombus (Czech Republic, 1992; } \\
\text { V. Ptáček, pers. comm., 2002) }\end{array}$ & $\begin{array}{l}\text { Škapec (1992; does not include } \\
\text { Bombus) }\end{array}$ & \\
\hline SVK & $\begin{array}{l}\text { All Bombus (Slovak Republic, 1999; } \\
\text { R. Chlebo, pers. comm., 2002) }\end{array}$ & $\begin{array}{l}\text { Škapec (1992; does not include } \\
\text { Bombus) }\end{array}$ & \\
\hline HUN & $\begin{array}{l}\text { Only B. argillaceus (K. Szauter, pers. } \\
\text { comm., 2001; M. Sárospataki, pers. } \\
\text { comm., 2002) }\end{array}$ & $\begin{array}{l}\text { Rakonczay (1989; includes only } \\
\text { B. argillaceus \& B. fragrans) }\end{array}$ & \\
\hline POL & $\begin{array}{l}\text { All Bombus \& } 2 \text { species of Psithyrus } \\
\text { (Minister of Environment, 2004) }\end{array}$ & $\begin{array}{l}\text { Kosior (1992b; includes only } \\
\text { B. jonellus) }\end{array}$ & Banaszak (2002) \\
\hline
\end{tabular}

${ }^{*}$ Red List, a list of species with their Red List categories; Red Book, a comprehensive Red List assessment of each species.

The assessment of factors threatening the Bombini was based largely on expert opinion. Whilst there are disadvantages to the use of such subjective judgement it was, however, the only way to obtain proxy information of drivers of Bombini decline in the region. Although experts' opinions on the importance of factors such as hiking or road kills were diverse, they were consistent on the importance of the impacts of various aspects of large-scale farming schemes, similar to findings regarding the role of agricultural intensification (Banaszak, 1995) and habitat fragmentation and isolation (SteffanDewenter \& Tscharntke, 2002) in Bombini declines.

In the opinion of the experts surveyed the importation of alien pollinators is not important in the decline of the Bombini, although they noted the important role of competition with honey bees Apis spp.. Competition for floral resources occurs between Bombini and several introduced bee species (Goulson, 2003), and between wild bees and managed pollinators (Butz-Huryn, 1997; Steffan-Dewenter \& Tscharntke, 2000). Competition with honey bees has been identified as one of the factors contributing to the extinction or decline of bumble bee species in central England, along with predation, parasites, pesticides, and changes in vegetation structure (Williams, 1982). The latter was largely a result of intensification of agriculture: an acceleration of mechanization, drainage of pastures, and the increase in the size of fields by the removal of hedges. Intensification of agricultural practices are regarded as contributing to the decline of Bombini in the former East Germany, Belgium and Northern France (Williams, 1982).

Species richness amongst British bumble bees is influenced by the availability of food plants (Williams, 1989), and long-tongued and therefore more specialized bumble bees are more likely to have exhibited a decline (Goulson et al., 2005). For this reason introduction of non-native flowering plants may also contribute to pollinator loss (Brown \& Mitchell, 2001; Chittka \& Schürkens, 2001). There may be a synergistic effect of pressure from natural mortality factors and the increasing impact of anthropogenic effects on the Bombini. For example, the spread of diseases and parasites of Bombini has been identified as an important driver of pollinator loss (Watanabe, 1994). It is known that the nematode Sphaerularia bombi is capable of sterilizing gonads in $35-90 \%$ of wintering bumble bee females (Pouvreau, 1974).

The general conclusion from our analysis is that to halt population declines and species extinctions of Bombini it will be necessary, above all, to preserve aspects of traditional farming practices. The maintenance 
of habitat patchiness and limiting use of chemical preparations and heavy machinery are of key importance for the Bombini. Approaches to the conservation of Bombini differ significantly between countries, and in some countries of the region the protection afforded to this economically important group of insects is inadequate. Given their importance as pollinators it would be appropriate for all Bombini taxa to be afforded legal protection in all countries of the region, and for threatened taxa to be assessed for inclusion in the relevant national and regional Red Lists.

The growing evidence of pollinator loss in Europe has resulted in a number of initiatives to protect the Bombini. A recent European Union funded project, ALARM (Assessing LArge scale environmental Risks for biodiversity with tested Methods; Settele et al., 2005), focuses on risks consequent, amongst others, on loss of pollinators. As changes in agricultural practices are probably the main single factor affecting Bombini in the region, and as the majority of the countries are EU members, the implementation of the EU's Common Agricultural Policy is likely to have the greatest single impact upon pollinators in the near future. A prerequisite to halt pollinator loss in Europe is for this Policy to follow advice provided by results of scientific research. Unfortunately, pollinator loss is rarely perceived as a serious threat to biodiversity by the general public, and an increase in awareness of this issue is therefore also of great importance.

\section{Acknowledgements}

We thank the following specialists who made available their unpublished data: J. Neumayer (Austria), P. Rasmont (Belgium), M. Pavelka, A. Přidal, V. Ptáček and I. Valterova (Czech Republic), I. Calabuig, H.B. Madsen and R. Mortensen (Denmark), C. van Achterberg (Netherlands), M. Sárospataki and K. Szauter (Hungary), P. Westrich (Germany), R. Chlebo, J. Slamecka and V. Smetana (Slovakia), F. Amiet and S. Durrer (Switzerland), and T. Pawlikowski (Poland). We also thank three anonymous reviewers for their invaluable comments on an earlier version of this article. W. Król and W. Solarz were partly funded by EU project ALARM, No. GOCE-CT-2003-506675.

\section{References}

Aichorn, A.A. (1983) Zur Gefährdungssituation der Hummeln in Österreich (Bombus, Hymenoptera). In Rote Listen gefährdeter Tiere Österreichs. 1 (ed. J. Gepp), pp. 3-242. Im Auftrag des Bundes-ministeriums für Gesundheit und Umweltschutz, Wien, Austria.

Allen-Wardell, G., Bernhardt, T., Bitner, R., Burquez, A., Cane, J., Cox, P.A., Dalton, V., Feinsinger, P., Ingram, M., Inouye,
D., Jones, C.E., Kennedy, K., Kevan, P., Koopowitz, H., Medellin, R., Medellin-Morales, S., Nabhan, G.P., Pavlik, B., Tepedino, V., Torchio, P. \& Walker, S. (1998) The potential consequences of pollinator declines on the conservation of biodiversity and stability of crop yields. Conservation Biology, 12, 8-17.

Amiet, F. (1994) Rote Liste der gefährdeten Bienen der Schweiz. In Rote Listen der gefährdeten Tierarten der Schweiz (ed. P. Duelli), pp. 38-44. Hrsg. Bundesamt für Umwelt, Wald und Landschaft, BUWAL-Reihe Rote Listen, Bern, Switzerland.

Banaszak, J. (1993) Trzmiele Polski. WSP, Bydgoszcz, Poland.

Banaszak, J. (1995) Changes in Fauna of Wild Bees in Europe. Pedagogical University, Bydgoszcz, Poland.

Banaszak, J. (1996) Import trzmieli do Polski - korzyści i zagrożenia. Chrońmy Przyrodę Ojczysta, 52, 110-116.

Banaszak, J. (2000) A checklist of the bee species (Hymenoptera, Apoidea) of Poland, with remarks on their taxonomy and zoogeography: revised version. Fragmenta Faunistica, 43, 135-193.

Banaszak, J. (2002) Apoidea. In Red List of Threatened Animals in Poland (ed. Z. Głowaciński), pp. 80-87. Institute of Nature Conservation, Polish Academy of Sciences, Kraków, Poland.

Banaszak, J., Michalik, S., Fijał, J. \& Kosior, A. (1998) Wpływ sukcesji zbiorowisk nieleśnych na pszczołowate Apoidea rezerwatu leśno-stepowego Skołczanka. Pradnik Prace $i$ Materiały Muzeum Im. Prof. Władysława Szafera, 11-12, 223-250.

Beláková, A., Smetana, V. \& Valenčík, M. (1979) Výskyt niektorých zástupcov podčeladí Bombinae a Psithyrinae (Hymenoptera, Apoidea) na Slovensku. Biológia, 34, 637-644.

Blab, J., Nowak, E., Trautmann, W. \& Sukopp, H. (eds) (1984) Rote Liste der gefährdeten Tiere und Pflanzen in der Bundesrepublik Deutschland. 4. Erweiterte und bearbeitete Auflage. Kilda-Verlag, Greven, Germany.

Brown, B.J. \& Mitchell, R.J. (2001) Competition for pollination: effects of pollen of an invasive plant on seed set of a native congener. Oecologia, 129, 43-49.

Burd, M. (1994) Bateman's principle and plant reproduction: the role of pollen limitation in fruit and seed set. Botanical Review, 60, 83-139.

Butz-Huryn, V.M. (1997) Ecological impacts of introduced honey bees. The Quarterly Review of Biology, 72, 275-296.

Celary, W., Fijał, J., Kosior, A., Król, W., Solarz, W. \& Witkowski, Z. (2004) Changes in the species composition and range of Bombini (Apidae) in Cracow since the mid 1850s. Unpublished Report. Institute of Nature Conservation, Polish Academy of Sciences, Kraków, Poland.

Chittka, L. \& Schürkens, S. (2001) Successful invasion of a floral market. Nature, 411, 653.

Corbet, S.A., Williams, I.H. \& Osborne, J.L. (1991) Bees and the pollination of crops and wild flowers in the European Community. Bee World, 72, 47-59.

Czech Republic (1992) Law No. 114/1992 and Edict No. 395/1992. Praha, Czech Republic.

Dathe, H.H. \& Saure, C. (2000) Rote Liste und Artenliste der Bienen des Landes Brandenburg (Hymenoptera: Apidae). Beilege zum Heft 1. Naturschutz und Landschaftspflege in Brandenburg, 9, 1-35.

Dylewska, M. (1996) Nasze trzmiele. ODR, APW, Karniowice, Polska.

Gauld, I.D., Collins, N.M. \& Fitton, M.G. (1990) The biological significance and conservation of Hymenoptera in Europe. Nature and Environment Series, 44, 3-47. 
Gepp, J. (1983) Rote Listen gefährdeter Tiere Österreichs. 1. Im Auftrag des Bundes-ministeriums für Gesundheit und Umweltschutz, Wien, Austria.

Goulson, D. (2003) Effects of introduced bees on native ecosystems, Annual Review of Ecology, Evolution and Systematics, 34, 1-26.

Goulson, D., Hanley, M.E., Darvill, B., Ellis, J.S. \& Knight, M.E. (2005) Causes of rarity in bumble bees. Biological Conservation, $122,1-8$.

Herrera, C. \& Pellmyr, O. (eds) (2002). Plant-Animal Interactions. Blackwell Science, Malden, USA.

IUCN (2001) 2001 Categories and Criteria (version 3.1). IUCN, Gland, Switzerland [http://www.redlist.org/info/ categories_criteria2001.html, accessed 18 October 2004].

Kearns, C.A., Inouye, D.W. \& Waser, N.M. (1998) Endangered mutualisms: the conservation of plant-pollinator interactions. Annual Review of Ecology and Systematics, 29, 83-112.

Kosior, A. (1987) Impact of economic activity upon bumblebee Bombus Latr. population in the West Bieszczady Mts. Ochrona Przyrody, 45, 239-262.

Kosior, A. (1990) The bumble bees Bombus Latr. of the selected glades of the Tatra National Park. Studia Naturae, A, 34, 113-123.

Kosior, A. (1992a) Bumble bees (Bombus Latr.) and cuckoo bees (Psithyrus Lep.) in selected areas of the Hohe Tauern (Central Alps, Austria). Ochrona Przyrody, 50, 153-171.

Kosior, A. (1992b) Bombus jonellus (Kirby, 1802). Trzmiel tajgowy. In Polish Red Data Book of Animals (ed. Z. Głowaciński), pp. 300-302. PWRiL, Warszawa, Polska.

Kosior, A. (1992c) The influence of anthropogenic factors on the decline of bumble bees (Bombus Latr.) in Poland. In Natural Resources of Wild Bees in Poland (ed. J. Banaszak), pp. 145-152. Pedagogical University, Bydgoszcz, Poland.

Kosior, A. \& Nosek, A. (1987) Species composition and number of bumble bees Bombus Latr. in the areas influenced by the emissions from non-ferrous metal works in the Silesian Upland. Studia Naturae, A, 31, 81-99.

Kosior, A. \& Witkowski, Z. (1997) Stan przyrody ożywionej w rejonie zespołu zbiorników wodnych Czorsztyn-Niedzica i Sromowce Wyżne w trakcie piętrzenia (w 1997 r.). In Fauna trzmieli (Bombus Latr.) i trzmielców (Psithyrus Lep.) obszaru zbiornika Czorsztyńskiego na tle fauny obszarów sasiaduja cych (Pieniny Centralne, Pieniny Spiskie i pasmo Lubania) (ed. Z. Witkowski), pp. 1-2. Unpublished Report. Institute of Nature Conservation, Polish Academy of Sciences, Krakow, Poland.

Lukas, J. (2001) Cerveny (ekosozologicky) zoznam blanokridlovcov (Hymenoptera) Slovenska. In Cerveny zoznam rastlin a zivocichov Slovenska (eds D. Balaz, K. Marhold \& P. Urban), Ochrana Prirody, 20, 129-133.

May, J. (1959) Čmeláci v ČSR, jejich bionomie, chov a hospodářský význam. Sborník Akademie zemědělských věd ČSFR, Praha, Cechoslovakia.

Minister of Environment (2004) Decree on strictly protected animal species, 28 October. Dziennik Ustaw, 220, 15570-15582.

Móczár, M. (1957) Méhfélék Apidae. Hymenoptera 3. Fauna Hungariae, 19, 1-76.

Monsevičius, V. (1995) A check-list of wild bee species (Hymenoptera, Apoidea) of Lithuania with data to their distribution and bionomics. New and Rare for Lithuania Insect Species. Records and Descriptions of 1994-1995: 7-144.

Müller, H. (1881) Alpenblumen, ihre Befruchtung durch Insekten und ihre Anpassungen an dieselben. W. Engelmann, Leipzig, Germany.
Neumayer, J. \& Paulus, H.F. (1999) Ökologie alpiner Hummelngemeinschaften: Blütenbesuch,

Ressourcenaufteilung und Energiehaushalt. Stapfia, 67, 5-246.

NHM (Natural History Museum) (2005) Bombus Bumblebees of the World. Http:/ /www.nhm.ac.uk/research-curation/ projects/bombus/ [accessed 10 August 2006].

Pagliano, G. (1994) Catalogo degli Imenotteri Italiani. IV. (Apoidea: Colletidae, Andronidae, Megachilidae, Anthophoridae, Apidae). Memorabilia Societatis Entomologica Italiana, 72, 331-467.

Peeters, T.M.J., Raemakers, I.P. \& Smit, J. (1999) Voorlopige atlas van de Nederlandse bijen (Apidae). EIS, Leiden, Nederland.

Peeters, T.M.J. \& Reemer, M. (2003) Bedreigde en verdwenen bijen in Nederland (Apidae s.l.). Basisrapport met voorstel voor de Rode lijst. EIS-Nederland, Leiden, Netherlands.

Pouvreau, A. (1974) Les ennemis des bourdons. II. Organismes affectant les adultes. Apidologie, 5, 39-62.

Prridal, A. (2004) Checklist of the bees in the Czech Republic and Slovakia with comments on their distribution and taxonomy. (Insecta: Hymenoptera: Apoidea). Acta Universitatis Agriculturae et Silviculturae Mendelianae Brunensis, 52, 29-65.

Přidal, A. \& Tkalců, B. (2003) Records of two bumblebee species new for the Czech Republic and Slovakia (Hymenoptera: Apoidea: Bombini). Entomofauna, 24, 317-329.

Rakonczay, Z. (1989) Red Data Book of Animals in Hungary. Akadémia Kiadó, Budapest, Hungary.

Rasmont, P. (1983) Catalogue commenté des Bourdons de la région ouest-paléarctique (Hymenoptera, Apidae, Bombinae). Notes fauniques de Gembloux, 7, 1-72.

Rasmont, P. (1995) How to restore the Apoid diversity in Belgium and France? Wrong and right ways, or the end of protection paradigm! In Changes in Fauna of Wild Bees in Europe (ed. J. Banaszak), pp. 53-63. Pedagogical University, Bydgoszcz, Poland.

Rasmont, P., Ebmer, A., Banaszak, J. \& van der Zanden, G. (1995) Hymenoptera Apoidea Gallica. Liste taxonomique des abeilles de France, de Belgique, de Suisse et du Grand-Duché de Luxemburg. Bulletin de la Société Entomologique de France, 100, 1-98.

Rasmont, P., Leclercq, J., Jacob-Remacle, A., Pauly, A. \& Gaspar, C. (1993) The faunistic drift of Apoidea in Belgium. In Bees for Pollination (ed. E. Bruneau), pp. 65-87. Commission of the European Communities, Brussels, Belgium.

Rasmont, P. \& Mersch, P. (1988) Première estimation de la dérive faunique chez les bourdonsde la Belgique (Hymenoptera: Apidae). Annales de la Société Royale Zoologique de Belgique, 118, 141-147.

Razowski, J. (1985) Changes in the Lepidopterous fauna of Cracov, Poland. Nota Lepidopterologica, 8, 65-68.

Reinig, W.F. (1981) Synopsis der in Europa nachgewiesenen Hummel- und Schmarotzerhummelarten. Spixiana, 4, 159-164.

Riess, W., Roth, H.M. \& Nitsche, G. (1976) Rote Liste bedrohter Tiere in Bayern (Wirbeltiere und Insekten). Fassung 1. Bayerisches Landesamt für Umweltschutz. Schriftenreihe für Naturschutz und Landschaftspflege, 7, 5-38.

Sárospataki, M., Novák, J. \& Molnár, V. (2003) Hazai poszméh és álposzméhfajok (Hymenoptera: Apidae, Bombus és Psithyrus) UTM térképezése és az adatok természetvédelmi felhasználhatósága. Allattani Közlemények, 88, 1-24.

Schwartz, M., Gusenleitner, F., Westrich, P. \& Dathe, H.H. (1996) Katalog der Bienen Österreichs, Deutschlands und der Schweiz (Hymenoptera, Apidae). Entomofauna, Supplement 8, 1-398. 
Settele, J., Hammen, V.C., Hulme, P.E., Karlson, U., Klotz, S., Kotarac, M., Kunin, W.E., Marion, G., O'Connor, M., Petanidou, T., Peterson, K., Potts, S.G., Pritchard, H., Pysek, P., Rounsevell, M., Spangenberg, J., Steffan-Dewenter, I., Sykes, M.T., Vighi, M., Zobel, M. \& Kuhn, I. (2005) ALARM: Assessing LArge scale environmental Risks for biodiversity with tested Methods. GAIA - Ecological Perspectives in Science, Humanities, and Economics, 14, 69-72.

Siła-Nowicki, M. (1864) Przyczynek do owadniczej fauny Galicyi. Drukarnia Uniwersytecka, Kraków, Poland.

Škapec, L. (1992) Ĉervení Kniha ochrozených a vzácnych druhov rastlin a živočichov ĈSFR. 3. Bezstavovce. Príroda, Bratislava, Slovakia.

Slovak Republic (1999) Supplement of the Edict 93/1999. Bratislava, Slovakia.

Starzyk, J.R. \& Kosior, A. (1985) Effect of hiking on the entomofauna of the Bieszczady National Park and the surrounding area. Parki Narodowe i Rezerwaty Przyrody, 6, 93-100.

Steffan-Dewenter, I. \& Tscharntke, T. (2000) Resource overlap and possible competition between honey bees and wild bees in central Europe. Oecologia, 122, 288-296.

Steffan-Dewenter, I. \& Tscharntke, T. (2002) Insect communities and biotic interactions on calcareous grasslands - a mini review. Biological Conservation, 104, 275-284.

Śnieżek, J. (1893) O krajowych gatunkach trzmieli. Sprawozdanie Komisji Fizjograficznej, 29, 1-22.

Śnieżek, J. (1899) O krajowych gatunkach trzmielców. Sprawozdanie Komisji Fizjograficznej, 34, 86-95.

Tkalců, B. (1974) Bemerkenswerte Bienenfunde in der Tsc hechoslowakei (Hymenoptera: Apoidea). Acta Entomologica Bohemoslovaka, 71, 205-208.

Tkalců, B. (1999) Two species of bumblebees new for the Czech Republic (Hymenoptera: Apoidea). Sborník Př́rodovědného klubu v Uh. Hradišti, 4, 121-123.

van der Smissen, J. \& Rasmont, P. (1999) Bombus semenoviellus Skorikov 1910 eine für Westeuropa neue Hummelart (Hymenoptera: Bombus, Cullumanobombus). Bembix Bielefeld, 13, 21-24.

von Hagen, E. \& Aichhorn, A.A. (2003) Hummeln: bestimmen, ansiedeln, vermehren, schützen. Fauna Verlag, Nottuln, Germany.

Warncke, K. (1986) Die Wildbienen Mitteleuropas, ihre gültigen Namen und ihre Verbreitung. Entomofauna, Supplement 3 , $1-128$.

Warncke, K., Westrich, P., Preuss, G. \& Riemann, H. (1984) Rote Liste der Bienen (Apoidea). In Rote Liste der gefährdeten Tiere und Pflanzen in der Bundesrepublik Deutschland. 4. erweiterte und bearbeitete Auflage (eds J. Blab, E. Nowak, W. Trautmann \& H. Sukopp), pp. 50-52. Kilda-Verlag, Greven, Germany.

Watanabe, M.E. (1994) Pollination worries rise as honey bees decline. Science, 265, 1170

Wergin, J. (1977) Tiere in Gefahr. Naturschutz und Naturparke, 85, 40-42.

Westrich, P. (1990) Die Wildbienen Baden-Württembergs. Spezieller Teil: Die Gattungen und Arten. Verlag Eugen Ulmer, Stuttgart, Germany.

Westrich, P. \& Schmidt, K. (1985) Rote Liste der Stechimmen Baden-Württembergs (Hymenoptera Aculeata ausser
Chrysididae) (Stand 01. 01. 1985). Arbeitsblätter zum Naturschutz, 5, 35-42.

Westrich, P., Schwenninger, H.R., Dathe, H.H., Riehmann, H., Saure, C., Voith, J. \& Weber, K. (1998) Rote Liste der Bienen (Hymenoptera: Apidae) Deutschlands (Bearbeitungsstand: 1997). In Rote Liste gefährdeter Tiere Deutschlands (eds M. Binot, R. Bless, P. Boye, H. Gruttke \& P. Pretscher). Schriftenreihe für Landschaftspflege und Naturschutz, 55, 119-129.

Wierzejski, A. (1868) Przyczynek do fauny owadów błonkoskrzydłych (Hymenoptera). Sprawozdanie Komisji Fizjograficznej, 2, 108-120.

Wierzejski, A. (1874) Dodatek do fauny owadów błonkoskrzydłych (Hymenoptera). Sprawozdanie Komisji Fizjograficznej, 8, 253-273.

Williams, P.H. (1982) The distribution and decline of British bumble bees (Bombus Latr.). Journal of Apicultural Research, 21, 236-245.

Williams, P.H. (1988) Habitat use by bumble bees (Bombus spp.). Ecological Entomology, 13, 23-237.

Williams, P.H. (1989) Why are there so many species of bumble bees at Dungeness? Botanical Journal of the Linnean Society, 101, 31-44.

Williams, P.H. (1994) The dependence of crop pollination within the European Union on pollination by honey bees. Agricultural Zoology Reviews, 6, 229-257.

Williams, P.H. (2005) Does specialization explain rarity and decline among British bumble bees? A response to Goulson et al.. Biological Conservation, 122, 33-43.

Winter, R. (1994) Rote Liste der Wildbienen (Hymenoptera, Apoidea) Thueringens. Landschaftspflege und Naturschutz in Thueringen, 31, 86-90.

\section{Biographical sketches}

Andrzej Kosior's long-term interest in pollinators has resulted in an extensive database on the distribution and biology of bumble bees, cuckoo bees and butterflies, and he has a particular interest in the ecology and conservation of insect pollinators.

Waldemar Celary has studied the systematics of the wild bees of Central Europe for almost 20 years, and since 1998 he has also been conducting research on their biology.

Paweł Olejniczak is interested in the mathematical modeling of plant-insect relations.

Jan Fijał has studied the distribution of Hymenoptera, amphibians and reptiles in Poland, and has contributed to the Polish Red Data Books of vertebrates and invertebrates.

Wiesław Król's main interests are in database design and geographical information system analysis of species distributions.

Wojciech Solarz studies alien species invasions and the population ecology of birds.

Piotr Płonka is interested in the biology of ants and the management of protected areas. 